\title{
Intrasternal respiratory epithelium-lined foregut cyst
}

\author{
Sven Seiwerth, MD, ${ }^{a}$ Corinna Brüschke, MD, ${ }^{a}$ Juergen Wiens, MD, ${ }^{a}$ Helmut Ostertag, MD, ${ }^{a}$ and \\ Paolo Macchiarini, MD, PhD, ${ }^{\mathrm{b}}$ Hannover, Germany
}

$\mathrm{F}$ oregut or enterogenous cysts are the result of an abnormal budding or division of the primitive foregut and are usually found in adults. On the basis of histologic features and embryogenesis, they are classified into bronchogenic, esophageal, gastroenteric, and neuroenteric cysts and usually occur in the mediastinum or, less frequently, in the soft tissues of the chest wall. ${ }^{1}$ Intrasternal foregut cysts lined by respiratory epithelium have never previously been described in the literature, and we present here the first case.

\section{Clinical Summary}

A 61-year-old woman was admitted because of the onset of moderate and recurrent feeling of retrosternal pressure and pain and a cystic lesion of unknown histologic type of the manubrium sterni growing partially into the superior ventral mediastinum up to the aortic arch. Results of physical examination were normal. Conventional tomography and thoracic computed tomography (Figure 1) showed a lesion of the manubrium sterni with impression and sclerosis of the compacta. The intraosseous part of the cyst had a 1 - to 2 -cm wide connection to a $5 \times 3$-cm retrosternal extraosseous part. The extraosseous lesion was attached to the aortic arch and was limited by a calcified, enhancing capsule. Calcification of the compacta and smooth bone rimming of the perforated part of the compacta indicated the presence of a long-lasting process. Conventional radiographs of the thorax from 2 and 10 years previously also showed a cystic sternal lesion, although smaller in size. Arteriography excluded an aortic aneurysm.

Because preoperative investigations were cytologically and histologically unrevealing, the patient underwent an open biopsy. This ruled out a malignancy. The decision was then made to perform an incomplete median sternotomy and a partial sternectomy including the manubrium sterni below its first upper centimeter and first $2 \mathrm{~cm}$ of the corpus sterni, cartilagos costalis of the first three ribs bilaterally, and internal vessels bilaterally but not both clavicles. The cyst was found adherent to the thymic fat tissue but not to the ventral pericardium or any other thoracic structures.

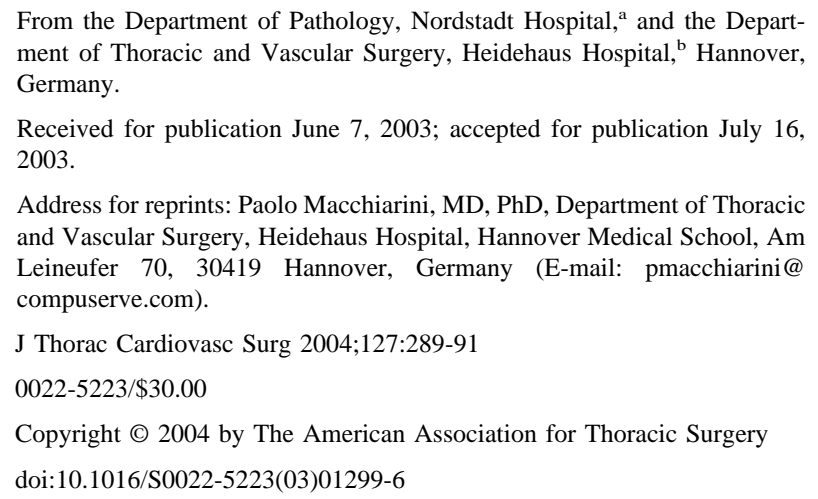

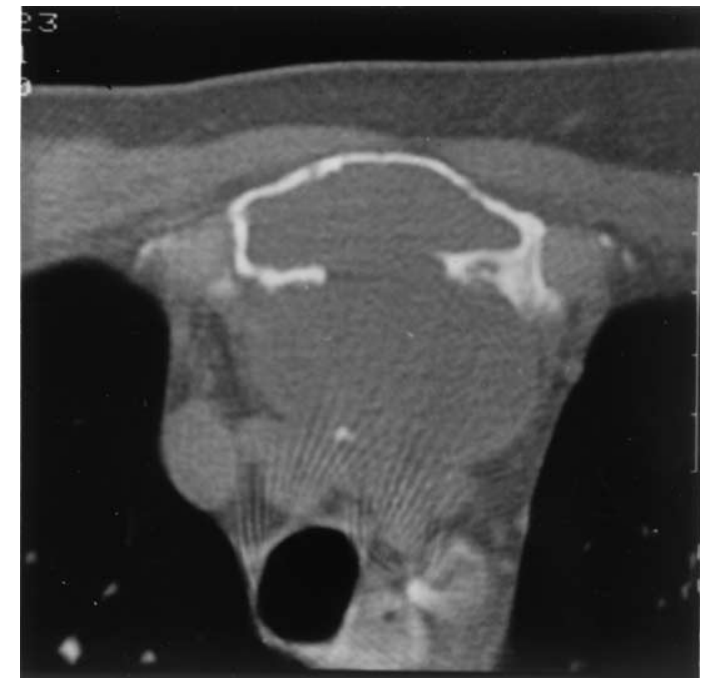

Figure 1. Chest computed tomography showing tumor destroying median posterior part of sternal bone, bulging into extraosseous soft tissue up to aortic arch. Mean density of retrosternal lesion is 37 Hounsfield units, corresponding to density of solid tissue, protein-rich fluid, or old hemorrhage.

The anterior chest wall was stabilized through the interposition of a polyglactin mesh (Vicryl; Ethicon Inc, Somerville, NJ) after adequate drainage of the pleural and mediastinal spaces. Both pectoralis major muscles were mobilized and joined together in the midline to add support to the wound, over which the skin was then closed in the usual fashion. The patient was extubated in the operating theater, and the postoperative recovery was uneventful.

The gross examination showed that the manubrium and corpus sterni contained a unilocular cyst $5.2 \mathrm{~cm}$ in length with a $1.7-\mathrm{cm}$ wide dorsal connection to an extraosseous tumor. The tumor appeared as a spherical bulging mass $5 \mathrm{~cm}$ in diameter that contained a yellow-green mucinous fluid (Figure 2). Histologically, the cyst consisted of a double layer of ciliated cuboid epithelium with an underlying thin fibrous stroma (Figure 3). Neither cartilage nor bronchial glands could be detected. Sternal cortex appeared normal. The extraosseous part of the cyst showed respiratory epithelium with more fibrous stroma. Focal regressive alterations of epithelium and stroma were noticed in the extraosseous part (metaplastic squamous epithelium, residues of hemorrhage, giant cells, proliferation of granulation tissue). Bone and periost reactions were absent. The resected thymic fat tissue was normal. The diagnosis of an intraosseous and extraosseous dysontogenetic foregut cyst was therefore made. Histochemical staining for mucopolysaccharides (periodic acid Schiff-Alcian blue stain) revealed unevenly distributed goblet cells in the parts of cyst wall lined by fully developed ciliated epithelium. Immunohistochemi- 


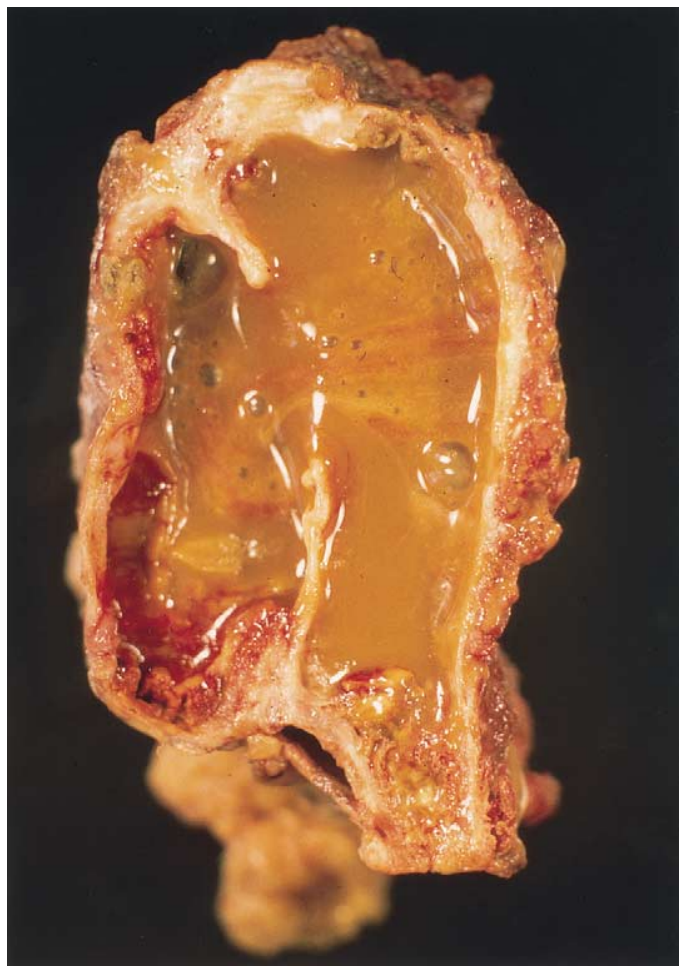

Figure 2. Resected manubrium shows dumbbell-shaped cyst, which extends into retrosternal tissue (left). Cyst is filled with yellow to green mucinous fluid.

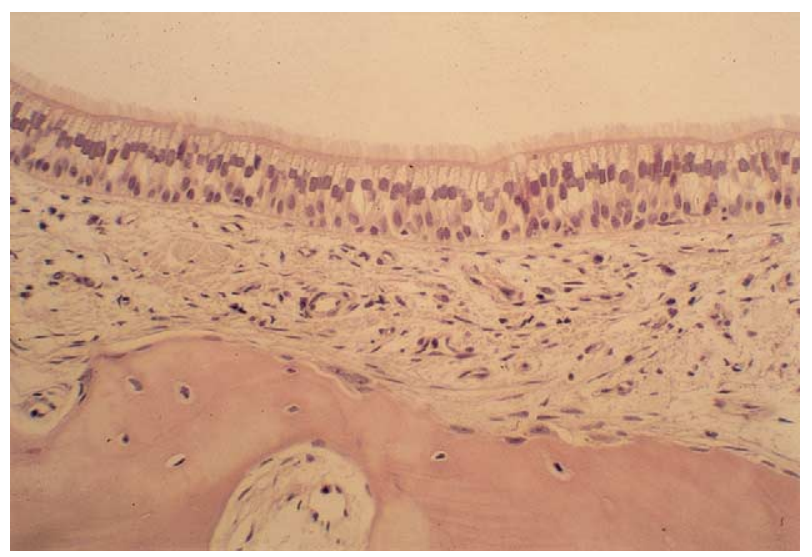

Figure 3. Histologic staining shows thin-walled bone cyst of manubrium and proximal corpus sterni, consisting of fibrous tissue with double layer of ciliated cylindric epithelium, typical of respiratory epithelium (hematoxylin and eosin; original magnification $200 \times$ ).

cal staining for cytokeratins was also performed and revealed that all tested samples expressed a slightly variable degree of immunoreactivity for cytokeratins 7,8 , and 19 , whereas staining for cytokeratin 20 remained uniformly negative. This staining pattern can be described as typical for ciliated respiratory epithelium, excluding any similarity to gastrointestinal tract columnar epithelium.

\section{Discussion}

Cystic structures lined by a different type of columnar epithelium originating from a different part of the primitive foregut are relatively rare anomalies. They are found in close contact with the tracheobronchial tree or the esophagus in the visceral compartment of the mediastinum.

In adults, the most common foregut cysts are the bronchogenic type, containing respiratory epithelium and cartilage or bronchial glands, and the esophageal type, containing respiratory epithelium and two or more smooth muscle layers. Because in the absence of specifically differentiated tissue other than respiratory epithelium the lesion is considered a foregut cyst, ${ }^{1}$ the cyst in our case corresponds to a foregut cyst.

During the 3rd to 4th week of gestation, the respiratory system develops from the primitive foregut as a ventral diverticulum with enlargement of two lung buds. If there is a third bud, it can separate as a foregut cyst. In the 9th week the fusion of the mesenchymal sternal bars begins. We consider that our patient's cyst originated from a supernumerary foregut bud that was included between the mesenchymal sternal bars during the 3rd through 8th weeks of gestation. The inclusion was incomplete, so that a part of the cyst wall remained extraosseous. Secretion of mucinous fluid caused an enlargement of the cyst, with secondary metaplastic squamous epithelium, hemorrhage, and fibrosis of the wall.

We only found a single case report of an intraosseous ciliated cyst, developing in the mandibular region after an autogenous bone graft from the nose to the chin when nasal respiratory epithelium was implanted traumatically into the chin. ${ }^{2}$ In our case report, the cyst was already detectable on a 10-year-old chest radiograph, and the patient's history did not include sternal injury. Teratoma, thymoma, and gastroenteric or pericardial cysts were ruled out because we found no tissue derived from more than two germ cell layers, no lymphatic tissue, no gastric or duodenal mucosa, and no flat mesothelial lining, respectively. ${ }^{3}$ As with most other visceral cystic lesions, foregut cysts must be completely resected because most will become symptomatic; complications may arise from size, fistulization, infection, and possibly even malignant alteration, and, last but not least, because operation on symptomatic cysts tends to be more complex. ${ }^{4}$ Moreover, partial excision or needle aspiration of the contents may cause recurrence.

Conclusion. We report here the first case in the literature of foregut cysts lined by respiratory-epithelium developing from a bony structure of the chest wall. This lesion was successfully managed with a partial sternectomy.

\section{References}

1. Salyer DC, Salyer WR, Eggleston JC. Benign developmental cysts of the mediastinum. Arch Pathol Lab Med. 1977;101:136-9.

2. Petkar M, Vaideeswar P, Deshpande JR. Surgical pathology of cystic lesions of the mediastinum. J Postgrad Med. 2001;47:235-9. 
3. Shimosato Y, Mukai K. Tumors of the mediastinum. In: Rosai J, Sobin LH, editors. Atlas of tumor pathology. No. 21. Washington, DC: Armed Forces Institute of Pathology; 1997. p. 40.
4. Bailey PV, Tracy T, Connors RH, deMello D, Lewis JE, Weber TR. Congenital bronchopulmonary malformations: diagnostic and therapeutic considerations. J Thorac Cardiovasc Surg. 1990;99:597-603.

\title{
Mediastinal growing teratoma syndrome after cisplatin-based chemotherapy and radiotherapy for intracranial germinoma
}

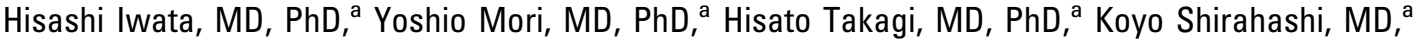 \\ J̧un Shinoda, MD, PhD, ${ }^{b}$ Kuniyasu Shimokawa, MD, PhD, ${ }^{c}$ and Hajime Hirose, MD, PhD, ${ }^{a}$ Gifu, Japan
}

$\mathrm{N}$ onseminomatous germ cell tumor (NSGCT) has been treated with cisplatin-based chemotherapeutic regimens. In cases of good response of tumor to chemotherapy, elevated tumor markers such as alpha-fetoprotein (AFP) and human chorionic gonadotropin (hCG) return to normal with tumor shrinkage. However, a small proportion of cases demonstrate tumor growth during or after the treatment. This distinctive situation was first described for testicular NSGCT by Logothetis and colleagues ${ }^{1}$ and was designated the growing teratoma syndrome (GTS). In this report we describe GTS of the mediastinum after cisplatin-based chemotherapy and radiotherapy for intracranial germ cell tumor.

\section{Clinical Summary}

A 20-year-old man with a rapidly growing mediastinal tumor was referred to our department. One month before, tumors in the pineal and the suprasellar regions had been found, with bitemporal hemianopia. Results of routine blood chemistry and coagulation studies were normal; however, serum AFP and hCG were increased to 447 $\mathrm{ng} / \mathrm{mL}$ and $240 \mathrm{ng} / \mathrm{ml}$, respectively. Serum carcinoembryonic antigen level was normal. The patient underwent partial resection of the suprasellar lesion for pathologic diagnosis. The pathologic diagnosis in the lesion was germinoma. Thereafter, the patient underwent chemotherapy consisting of ifosfamide, cisplatin, and etoposide followed by 20 Gy local irradiation. The intracranial lesions completely disappeared. Serum AFP and hCG were decreased to within normal limits.

After these therapies, a chest roentgenogram accidentally disclosed a mass located in the mediastinum. Chest computed tomog-

From First Department of Surgery, ${ }^{a}$ Department of Neurosurgery, ${ }^{\mathrm{b}}$ and Department of Laboratory Medicine, ${ }^{\mathrm{c}}$ Gifu University School of Medicine, Gifu, Japan.

Received for publication June 19, 2003; accepted for publication July 10, 2003.

Address for reprints: Hisashi Iwata, MD, PhD, First Department of Surgery, Gifu University School of Medicine, 40 Tsukasa-machi, Gifu City, Gifu 5008705, Japan (E-mail: ihisashi@cc.gifu-u.ac.jp).

J Thorac Cardiovasc Surg 2004;127:291-3

$0022-5223 / \$ 30.00$

Copyright $\odot 2004$ by The American Association for Thoracic Surgery

doi:10.1016/S0022-5223(03)01300-X raphy showed a round and lobulated mass in the anterosuperior mediastinum. The size of the mass rapidly increased (Figure 1). Tumor doubling time was calculated at 34.3 days. The tumor showed no response to $20 \mathrm{~Gy}$ irradiation. Gallium scintigraphy showed a slightly intense mediastinal enhancement region and no other lesion outside the chest. Histologic examination of a percutaneous needle biopsy specimen showed necrotic and fibrous connective tissue.

A median sternotomy was performed. The tumor was encapsulated in the thymic tissue. A complete resection was performed with negative margins.

On pathologic examination, the specimen of the mediastinal tumor was characteristic of mature teratoma. The tumor consisted of mature squamous epithelium with immature glandular structures. Immature structure tested immunohistochemically positive for carcinoembryonic antigen and keratin but negative for AFP and hCG (Figure 2, A). However, the immature component was less than $10 \%$ of the tumor. Retrospective pathologic examination in the intracranial lesion resected before chemoradiotherapy revealed pure germinoma with negative results of immunohistochemical testing for AFP and hCG (Figure 2, B).

The postoperative course was uneventful. At 8-month followup, the patient is doing well with no evidence of disease.

\section{Discussion}

Mediastinal germ cell tumors are heterogeneous groups of benign and malignant neoplasm thought to originate from primitive germ cells misplaced in the mediastinum. ${ }^{2}$ Teratoma, the most common mediastinal germ cell tumor, is classified as either mature teratoma, well-differentiated histologically and containing less than $10 \%$ fetal tissue, or immature teratoma, containing more than $10 \%$ fetal tissue. ${ }^{3}$

The definition of GTS encompasses three criteria: first, normalization of previously elevated serum tumor marker AFP or hCG, second, an increase in tumor size during or after chemotherapy given for NSGCT, and third, the absence of any NSGCT component other than mature teratoma in the tumor resected. ${ }^{1}$ The incidence of GTS among patients with metastatic NSGCT is between $1.9 \%$ and $7.6 \% .^{1,4}$

In our case, serum AFP and hCG levels had returned to normal after the chemoradiotherapy for intracranial lesions. The residual mediastinal tumor was disclosed accidentally and grew after chemoradiotherapy. The specimen of the mediastinal tumor was his- 